\title{
Optimal Generation Scheduling of Power System for Maximum Renewable Energy Harvesting and Power Losses Minimization
}

\author{
Bounthanh Banhthasit $^{1}$, Chaowanan Jamroen ${ }^{2}$, Sanchai Dechanupaprittha ${ }^{3}$ \\ ${ }^{1,3}$ Department of Electrical Engineering, Kasetsart University, Bangkok, Thailand \\ ${ }^{2}$ Division of Instrumentation and Automation Engineering Technology, \\ King Mongkut's University of Technology North Bangkok, Rayong Campus, Thailand
}

\begin{tabular}{l} 
Article Info \\
\hline Article history: \\
Received Sep 5, 2017 \\
Revised Mar 4, 2018 \\
Accepted Apr 2, 2018 \\
\hline
\end{tabular}

Keyword:

Distributed generation

Energy storage system

Generation scheduling

Particle swarm optimization

Power losses

Renewable energy harvesting

\begin{abstract}
This paper proposes an optimal generation scheduling method for a power system integrated with renewable energy sources (RES) based distributed generations (DG) and energy storage systems (ESS) considering maximum harvesting of RES outputs and minimum power system operating losses. The main contribution aims at economically employing RES in a power system. In particular, maximum harvesting of renewable energy is achieved by the mean of ESS management. In addition, minimum power system operating losses can be obtained by properly scheduling operating of ESS and controllable generations. Particle Swam Optimization (PSO) algorithm is applied to search for a near global optimal solutions. The optimization problem is formulated and evaluated taking into account power system operating constraints. The different operation scenarios have been used to investigate the effective of the proposed method via DIgSILENT PowerFactory software. The proposed method is examined with IEEE standard 14-bus and 30-bus test systems.
\end{abstract}

Copyright $\odot 2018$ Institute of Advanced Engineering and Science. All rights reserved.

\section{Corresponding Author:}

Bounthanh Banhthasit,

Department of Electrical Engineering,

Kasetsart University,

50, Ngam Wong Wan Rd, Lat Yao, Chatuchak, Bangkok, 10900, Thailand.

Email: banhthasit.b@gmail.com

\section{INTRODUCTION}

Development of renewable energy sources (RES) offers a remarkable benefit to sustainable power generation in all electric power system with low environmental pollution emissions compared with conventional energy sources based power generation. The most important aspect of renewable power generation, well known as distributed generation (DG), is almost zero fuel cost. However, DG connected existing power system is raising concerns on complex problems of system operation and control. Basically, the power flow in transmission lines will be changed in direction significantly causing undesired conditions of power losses in the power system. Although DG can additionally increase energy efficiency and enhance the capability of power system, it can adversely impact on power losses due to the change of unidirectional power flow to bidirectional power flow without appropriate power management [1]. Typically, DG is operated at rated capacity to achieve economical investment aspect, whereas it may lead to high level of power losses and critical voltage profiles in the power system. Therefore, generation scheduling method plays a significant factor for power management eliminating power losses and complexity of system operation problems.

Since power losses have been studied and presented in numerous aspects, some novel optimal power flows have been traditionally used in power losses reduction aspect [2]. Network reconfiguration was used for physically altering a network topology that aimed at change of the network power flow to reduce power 
losses in a power system [3]-[4]. Transmission expansion planning aspect could be employed to improve network losses considering reactive power [5]. Interconnection planning of microgrid has been discussed for enhancing power losses and reliability [6]. In distribution system, power losses are a significant issue due to high $\mathrm{R} / \mathrm{X}$ ratio. DG and capacitor locations were proposed for power losses reduction and ameliorating voltage profiles in distribution system [7]. In addition, switching devices allocation was analyzed in order to gain power loss reduction as well as service restoration [8]. Moreover, various methods were proposed in an area of DG units siting and sizing [9]-[11].

Although power loss minimization has been studied extensively, this aspect is still attracted the numerous research interests. Especially, the generation scheduling or power management based on power losses minimization has also been focused. For power management in distribution system, operational scheduling has been presented for overall benefit maximization and loss minimization based on electrical vehicles integrated system [12], [13]. Real-time energy management strategy was proposed considering operational constraints and power flow direction in microgrid [14], [15]. Demand response and renewable energy generation were considered for minimizing power loss using heuristic algorithm [16], [17]. Energy storage system (ESS) integrated microgrid was investigated to improve the loss minimization based on intermittent DG [18], [19].

This paper concentrates on an optimal generation scheduling method of power system considering maximum renewable energy harvesting and power loss minimization. The proposed method determines the significant variable of RES based power generation employing DG and ESS. However, DG accommodation and sizing cannot be adjusted in practical situation due to the capability constraints and economic benefit of a producer. Especially, DGs are always located at a specific areas that is uncontrollable factor. Although maximum DG outputs are expected for the producers, it can increase the power losses in the power system. Accordingly, DG output powers are managed within a proper way of maximum renewable energy harvesting. Then sufficient power will be supplied the loads while excessive power will be stored in ESS. The proposed method is investigated on IEEE standard 14-bus and 30-bus test systems via DIgSILENT Powerfactory software. Moreover, the proposed method is compared with an existing scheduling method to evaluate an effectiveness and confirm a superior performance.

\section{MATHEMATICAL MODEL}

The optimal generation scheduling problem for maximizing renewable energy harvesting from DG dispatch and minimizing power losses can be formulated and presented in (1) and (2) respectively.

$$
\begin{aligned}
& \text { Maximize } f_{1}=\max \left(P_{D G \text { dispatch }}\right) \\
& \text { Minimize } f_{2}=\min \left(P_{\text {Loss line }}\right)
\end{aligned}
$$

where $f_{1}$ and $f_{2}$ are the proposed objective functions. $P_{D G \text { dispatch }}$ is the renewable energy harvesting from DG dispatch in the power system (MW). $P_{\text {Loss line }}$ is the power loss in a transmission line (MW).

\subsection{Renewable energy harvesting model}

In practical aspect, DGs are always operated at the maximum rated power output ( $\left.P_{D G \text { output }}\right)$. This aspect may lead undesired conditions of power losses in the power system. On the other word, the utilities cannot directly control the power injected by DG. Therefore, this paper focuses on the renewable energy harvesting consisting of a DG dispatch $\left(P_{D G \text { dispatch }}\right)$ and an excessive power $\left(P_{\text {storage }}\right)$ between DG dispatch and maximum rated power output. The excessive power will be stored in ESS. The renewable energy harvesting function is given as follows:

$$
P_{D G \text { dispatch }}=P_{D G \text { output }}-P_{\text {storage }}
$$

As the excessive power, which highly links with the power loss in ESS, is stored in ESS. The electric energy storage system (ESS) loss can therefore be detailed consisting of battery loss and converter loss [20]. Thus the ESS loss can be calculated as follows:

$$
P_{\text {Loss ESS }}=P_{\text {Lossbattery }}+P_{\text {Lossconverter }}
$$




$$
\begin{aligned}
& P_{\text {Loss battery }}=I_{\text {battery }}^{2} \times R_{\text {battery }} \\
& P_{\text {Loss converter }}=P_{s b}+\left(k \% \times P_{\text {storage }}\right)
\end{aligned}
$$

where $P_{\text {Loss battery }}$ and $P_{\text {Loss converter }}$ are the battery loss and converter loss, respectively. $R_{\text {battery }}$ is the internal resistance of battery. $I_{\text {battery }}$ is the charging current related to the amount of power stored in $\operatorname{ESS}\left(P_{s t o r a g e}\right) . P_{s b}$ is the constant standby loss according to the power consumed by control platform, gate drivers, display, transducers and cooling fans. $k \%$ is the percentage of semiconductors and filter losses.

However, this paper considers the direct relationship of the maximum renewable energy harvesting. Hence, ESS loss is particularly assumed to be a variable of $P_{\text {storage }}$ instead of $I_{\text {battery }}$. As the $P_{\text {storage }}$ has been represented in (3) which significantly affects on the power loss of ESS. Consequently, the ESS loss can be assumed into a stored power in ESS and efficiency of $\operatorname{ESS}(\eta)$ that is provided as follows:

$$
\begin{aligned}
& P_{\text {storage }}=P_{D G \text { output }}-P_{D G \text { dispatch }} \\
& P_{\text {Loss ESS }}=(1-\eta) P_{\text {storage }}
\end{aligned}
$$

\subsection{Power loss in line model}

In order to obtain the power loss in line of the power system, the generalized power flow equation is employed in this paper to calculate the power losses. The power flow equation, deals with steady-state analysis related to real power and reactive power [21], can be expressed as follows:

$$
\begin{aligned}
& S_{i}=P_{i}+j Q_{i} \\
& P_{i}=\sum_{k=1}^{n}\left|V_{i}\right|\left|V_{k}\right|\left|Y_{i k}\right| \cos \left(\theta_{i}-\theta_{k}-\alpha_{i k}\right), \quad i=1,2, \ldots, \mathrm{n} \\
& Q_{i}=\sum_{k=1}^{n}\left|V_{i}\right|\left|V_{k}\right|\left|Y_{i k}\right| \sin \left(\theta_{i}-\theta_{k}-\alpha_{i k}\right), \quad i=1,2, \ldots, \mathrm{n}
\end{aligned}
$$

where $S_{i}, P_{i}$ and $Q_{i}$ are the net apparent power, active power and reactive power injections to bus $i$, respectively. $n$ is the total number of buses in the system. $V_{i}$ and $V_{k}$ are the voltage magnitudes at buses $i$ and $k$, respectively. $\theta_{i}$ and $\theta_{k}$ are the voltage angles at buses $i$ and $k$, respectively. $Y_{i k}$ is the admittance magnitude between buses $i$ and $k . \alpha_{i k}$ is the phase angle of admittance between buses $i$ and $k$. This paper merely considers the power losses in lines with respect to active part from a branch conductance $\left(g_{i k}\right)$ between buses $i$ and $k$ that can be described as follows:

$$
P_{\text {Loss line } e_{k}}=g_{i k}\left[V_{i}^{2}+V_{k}^{2}-2 V_{i} V_{k} \cos \left(\theta_{i}-\theta_{k}\right)\right]
$$

\subsection{Objective function formulation}

As Section 2.1 and 2.2, the maximum renewable energy harvesting is to maximize power dispatch of DG or minimize excessive power which can be represent minimum the power loss in ESS. The combination of Equations (1) and (2) is the objective function of the proposed method. Therefore, it can be expressed as follows:

$$
\operatorname{Min} P_{\text {Loss }}^{\text {Total }}=\sum_{i=1}^{N l} P_{\text {Lossline }, i}+\sum_{j=1}^{N s t} P_{\text {Loss ESS }, j}
$$

where $P_{\text {Loss line, } i}$ is the power loss in line $i, P_{\text {Loss ESS }, j}$ is the ESS loss at location $j . N l$ and $N s t$ are the total number of lines and the total energy storage locations, respectively. 


\subsection{Operational constraints}

\subsubsection{Power flow constraint}

The power flows across each line from any two buses $i$ and $j$ must be remained within the maximum limit by maximum current capacity of each line. The power flow constraint is given as follows:

$$
I_{i-j} \leq I_{i-j}^{\max }
$$

where $I_{i-j}$ is the current in the line between buses $i$ and $j . I_{i-j}^{\max }$ is the maximum current capacity of the line between buses $i$ and $j$.

\subsubsection{Generator constraints}

The generators in the system must be operated under the rated active and reactive power limits associated with the bus voltage. The voltage level must be also within maximum and minimum limits. The generator constraints can be provided as follows:

$$
\begin{aligned}
& P_{N}^{\min } \leq P_{N} \leq P_{N}^{\max } \\
& Q_{N}^{\min } \leq Q_{N} \leq Q_{N}^{\max } \\
& V_{N}^{\min } \leq V_{N} \leq V_{N}^{\max }
\end{aligned}
$$

where $P_{N}$ and $Q_{N}$ are the active and reactive power injection at generator bus $N$, respectively. $P_{N}^{\max }$ and $Q_{N}^{\max }$ are the maximum active and reactive powers of the generator $N$, respectively. $P_{N}^{\min }$ and $Q_{N}^{\min }$ are the minimum active and reactive powers of the generator $N$, respectively. $V_{N}$ is the bus voltage where a generator connected at bus $N . V_{N}^{\max }$ and $V_{N}^{\min }$ are the maximum and minimum operating voltage limits of a generator bus, respectively.

\subsubsection{Renewable distributed generation constraint} follows:

The renewable DG constraint is only considered the power output limit. The limit can be given as

$$
0 \leq P_{D G, N} \leq P_{D G, N}^{\max }
$$

where $P_{D G, N}$ is the active power dispatch from DG to bus $N . P_{D G, N}^{\max }$ is the maximum rated active power of each DG at bus $N$.

\subsubsection{Load constraints}

The general loads are distributed among the system while the loads should be operated in the voltage constraints as given in (19). Additionally, the load must be also operated under the voltage deviation $(V D)$ limit. $V D$ is represented as the difference of voltage between maximum and minimum voltage limits. The $V D$ can be expressed as given in (19) and (20).

$$
\begin{array}{ll}
V_{N}^{\min } \leq V_{N} \leq V_{N}^{\max } & N=1, \ldots, n . \text { bus no. } \\
V D_{i}=V_{i}^{\max }-V_{i}^{\min } & i=1, \ldots, m . \text { scinarios no. }
\end{array}
$$

where $V_{N}$ is the bus voltage where the load is connected at bus $N . V_{N}^{\max }$ and $V_{N}^{\min }$ are the maximum and minimum operating bus voltage limits, respectively. $V_{i}^{\max }$ and $V_{i}^{\min }$ are the maximum and minimum of system voltage at scenario $i$, respectively. 


\section{PROPOSED METHOD}

This proposed method applies PSO algorithm to explore the optimal solutions of generation scheduling for maximizing renewable energy harvesting and minimizing power losses in accordance with Section 2. The PSO algorithm is initialized with a set of random positions of particles (solutions) [22], [23]. Each particle will be executed to obtain the solution according to the objective function. Every iteration, the particles will be updated by following two important values. The first one is the best solution $\left(p_{\text {best }}\right)$ that links with cognitive factor. The other important value is tracked by the particle swarm optimizer in accordance with social factor. This value is a global best $\left(g_{\text {best }}\right)$. The particles are updated according to the $p_{\text {best }}$ and $g_{\text {best }}$ for obtaining the new positions and velocities using (21) and (22).

$$
\begin{aligned}
& v_{i}^{k+1}=v_{i}^{k}+c_{1} r_{1}\left(p_{\text {best }, i}^{k}-x_{i}^{k}\right)+c_{2} r_{2}\left(g_{\text {best }, i}^{k}-x_{i}^{k}\right) \\
& x_{i}^{k+1}=x_{i}^{k}+v_{i}^{k+1}
\end{aligned}
$$

where $v_{i}^{k}$ is the velocity of a particle $i$ at iteration $k . x_{i}^{k}$ is the position of a particle $i$ at iteration $k . p_{b e s t, i}^{k}$ is the personal best position of the particles. $g_{\text {best }, i}^{k}$ is the global best position of the particles. $c_{l}$ and $c_{2}$ are positive acceleration constants. $r_{l}$ and $r_{2}$ are the random values from uniform distribution.

Although the PSO algorithm is initialized with the random positions of particles $x$ to search the best position for obtaining the optimal solutions with respect to the proposed objective function, the swam can be determined as the multidimensional variables according to a number of designated variables in the power system. Therefore, the sets of particles $(X)$ and velocities $(V)$ are associated with a number of variables $(n)$ and number of particles $(m)$ that can be presented in (23) and (24). This paper considers 3 variables consisting of the conventional power generation $\left(P_{N}\right)$, voltage at generation bus $\left(V_{N}\right)$ and renewable distributed generation $\left(P_{D G \text { dispatch }}\right)$. The flowchart of the proposed method is represented in Figure 1.

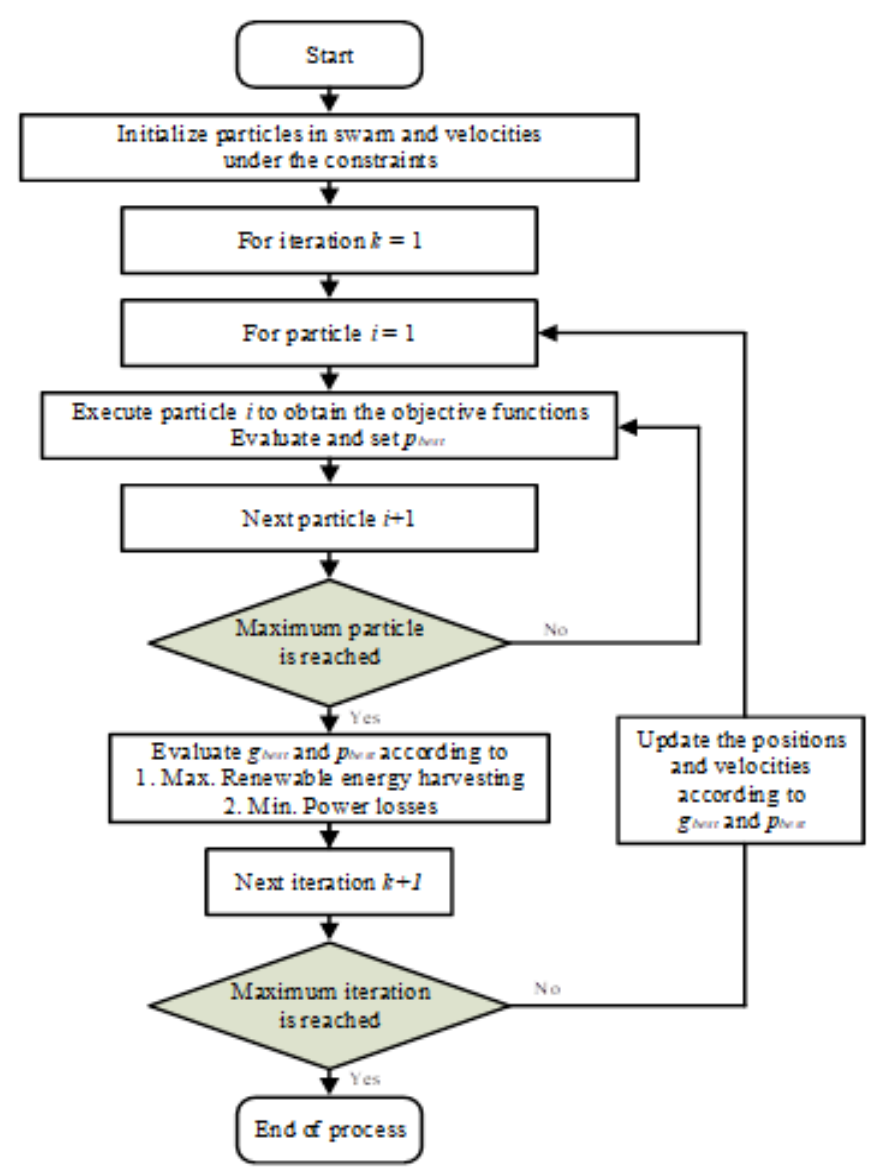

Figure 1. Flowchart of PSO based proposed method 


$$
\begin{aligned}
& X=\left[\begin{array}{cccc}
x_{1,1} & x_{1,2} & \ldots & x_{1, n} \\
x_{2,1} & x_{2,2} & \ldots & x_{2, n} \\
\vdots & \vdots & \vdots & \vdots \\
x_{m 1} & x_{m, 2} & \ldots & x_{m, n}
\end{array}\right] \\
& V=\left[\begin{array}{cccc}
v_{1,1} & v_{1,2} & \ldots & v_{1, n} \\
v_{2,1} & v_{2,2} & \ldots & v_{2, n} \\
\vdots & \vdots & \vdots & \vdots \\
v_{m, 1} & v_{m, 2} & \ldots & v_{m, n}
\end{array}\right]
\end{aligned}
$$

\section{CASES FOR COMPARISON}

In this section, two cases are performed for comparative study in order to exploit the capability of the proposed method. According to the objective function in Section 2, the study was designed in DIgSILENT Program Language (DPL). Furthermore, all cases are operated under the designated operational constraints as described in Section 2. The performed cases can be detailed as follows:

\subsection{Line loss minimization (case1)}

The generation units (conventional generation units and DG units) in the system will be searched to receive the optimal power dispatches considering solely power losses minimization. The power loss minimizing is considered the power losses in transmission lines under the operational constraints. Additionally, the search condition is regarded to voltage deviation. Therefore, the optimal solutions of case 1 $\left(x_{1}\right)$ can be defined according to the designated variables including conventional generation power $\left(P_{N}\right)$, voltage level $\left(V_{N}\right)$, and DG power $\left(P_{D G \text { dispatch }}^{\text {opt. }}\right)$ that can be provided as follows:

$$
\begin{aligned}
& x_{1}=\left[\begin{array}{lll}
P_{N} & V_{N} & P_{D G \text { dispatch }}^{\text {opt.1 }}
\end{array}\right]^{T} \\
& P_{D G \text { dispatch }}^{\text {opt. }} \in\left[0, P_{D G \text { output }}\right]
\end{aligned}
$$

\subsection{Maximum DG output dispatch (case2)}

The maximum renewable energy harvesting is the main objective function of this case. The method will be explored the optimal point of conventional generations and maximizing renewable energy harvesting from DG units. The search space of DG power is consistent and recalled from case1. However, the optimal renewable DG dispatch must to search within the DG limit and the previous DG power dispatch. The optimal solution of case $2\left(x_{2}\right)$ can be provided as follows:

$$
\begin{aligned}
& x_{2}=\left[\begin{array}{lll}
P_{N} & V_{N} & P_{D G \text { dispatch }}^{\text {opt.2 }}
\end{array}\right]^{T} \\
& P_{D G \text { dispatch }}^{\text {opt.2 }} \in\left[P_{D G \text { dispatch }}^{\text {opt.1 }}, P_{D G \text { output }}\right]
\end{aligned}
$$

\section{SIMULATION RESULTS AND DISCUSSION}

\subsection{Test systems description}

The proposed method is tested in comparative study with the performed cases under IEEE standard 14-bus and 30-bus test systems. The generation units consist of the conventional generation units and renewable DG units according to the test systems. The ESSs are installed in each location of renewable DG unit to collect the excessive power. The available DG power output at each location is comprised of power energy available of DG dispatch and stored excessive power in ESS. The components data of the 14-bus and 30-bus test systems are detailed in Table 1 and Table 2, respectively. To assess the ESS losses, the efficiency of ESS is assumed to be $90 \%$ for all installed locations. 
Table 1. Detail of IEEE Standard 14-bus Test System

\begin{tabular}{llcc}
\hline No. & \multicolumn{1}{c}{ Type } & Bus & $\begin{array}{c}\text { Cap. } \\
{[\mathrm{MW}]}\end{array}$ \\
\hline 1 & Conventional gen. unit 1 & 1 & 750 \\
2 & Conventional gen. unit 2 & 2 & 600 \\
3 & Conventional gen. unit 3 & 3 & 400 \\
4 & Renewable DG unit 1 & 12 & 100 \\
5 & Renewable DG unit 2 & 10 & 100 \\
6 & Renewable DG unit 3 & 9 & 100 \\
7 & ESS unit 1 & 12 & \\
8 & ESS unit 2 & 10 & \\
9 & ESS unit 3 & 9 & \\
& & & \\
\hline
\end{tabular}

Table 2. Detail of IEEE Standard 30-bus Test System

\begin{tabular}{llcc}
\hline No. & \multicolumn{1}{c}{ Type } & Bus & $\begin{array}{c}\text { Cap. } \\
{[\mathrm{MW}]}\end{array}$ \\
\hline 1 & Conventional gen. unit 1 & 1 & 200 \\
2 & Conventional gen. unit 2 & 2 & 150 \\
3 & Conventional gen. unit 3 & 5 & 150 \\
4 & Conventional gen. unit 4 & 8 & 80 \\
5 & Conventional gen. unit 5 & 11 & 50 \\
6 & Conventional gen. unit 6 & 13 & 50 \\
7 & Renewable DG unit 1 & 5 & 50 \\
8 & Renewable DG unit 2 & 3 & 50 \\
9 & Renewable DG unit 3 & 9 & 50 \\
10 & ESS unit 1 & 5 & \\
11 & ESS unit 2 & 3 & \\
12 & ESS unit 3 & 9 & \\
\hline
\end{tabular}

\subsection{Simulation Results}

This paper considered the variation of each generated DG power output in a particular day as shown in Figure 2. In general, DGs are always generated at maximum rated capacity based on renewable energy resources in time interval of a day. The power dispatching of DGs is respected to power loss in line and power loss in energy storing process.

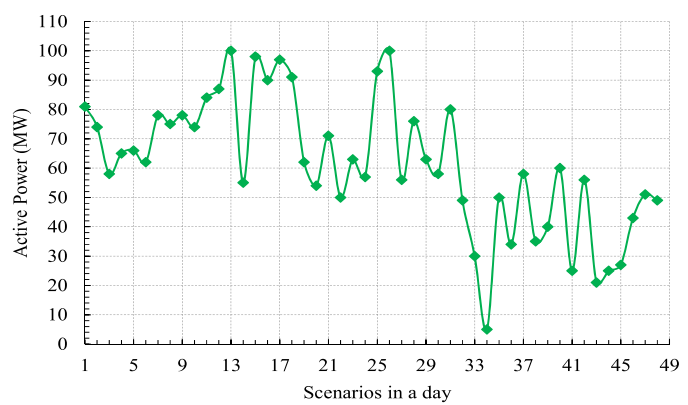

(a)

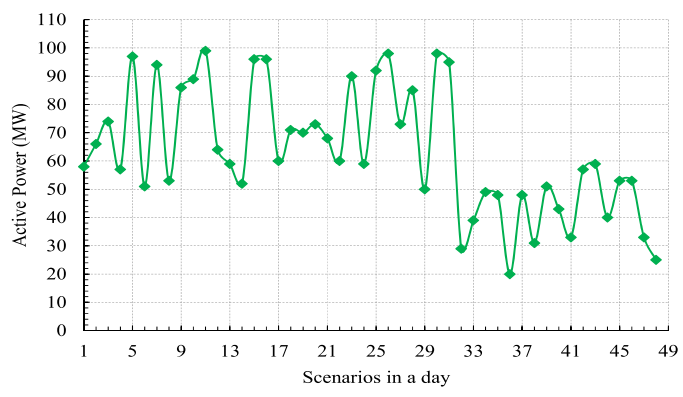

(b)

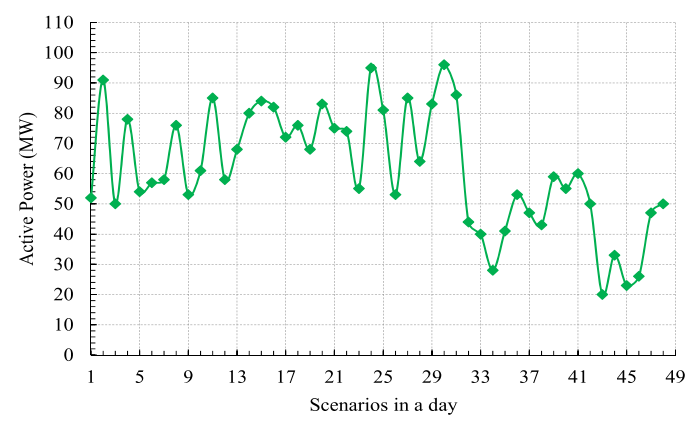

(c)

Figure 2. Power generation of renewable distributed generation in a particular day (a) DG unit 1

(b) DG unit 2 (c) DG unit 3

\subsubsection{IEEE standard 14-bus system}

The IEEE standard 14-bus test system was determined by 48 operating conditions in accordance with half an hour forecasting of load demand during a particular day in order to investigate the capability of the proposed method. The optimal generation scheduling was obtained by the proposed method compared with the performed cases as illustrated in Figure 3. Figure 3 demonstrated the load demand (MW), conventional generation (MW), maximum DG power output (MW) and DG dispatch (MW) in a particular day. The load demands were supplied by conventional generation together with DG to fulfill the mismatch power. The stored excessive powers in ESS were represented between the area of DG output curve and 
optimal DG dispatch. Since the excessive power influences the power loss in stored power process, thus the level of power loss in power storing process is presented by this area. The simulation results were summarized in Table 3.

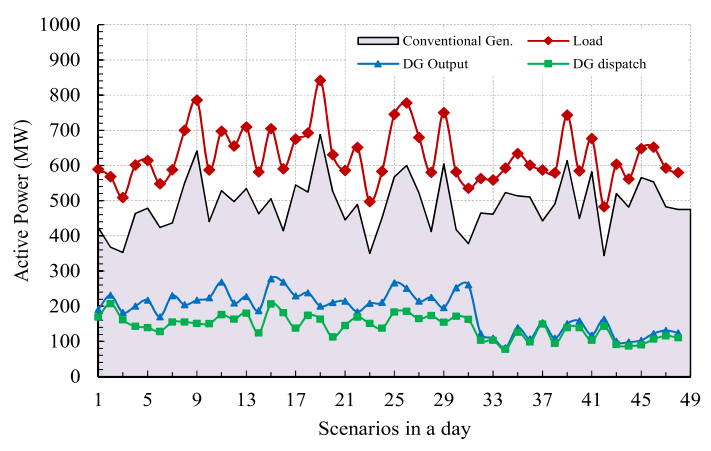

(a)

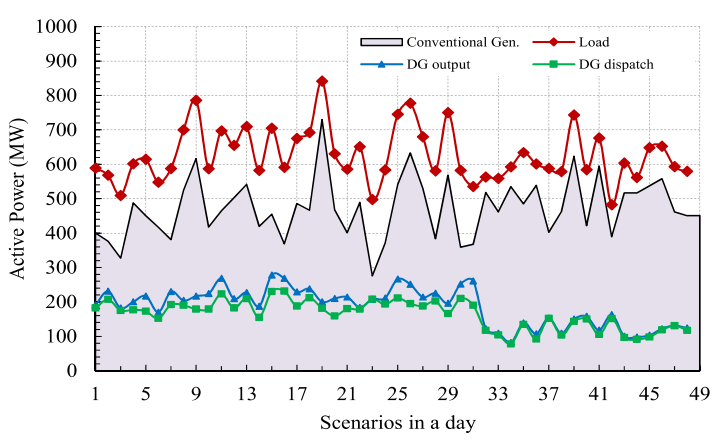

(b)

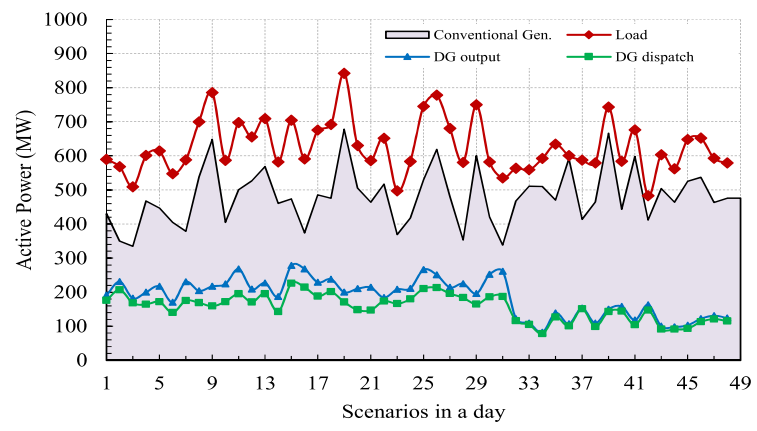

(c)

Figure 3. Optimal generation scheduling and load demand (a) case 1 (b) case 2 (c) Proposed method

Table 3. The IEEE 14-bus System Simulation Results

\begin{tabular}{lrrr}
\hline Parameters & Case1 & Case2 & Proposed method \\
\hline DG Harvesting (MW) & $6,840.9$ & $7,879.6$ & $7,509.9$ \\
Loss in lines (MW) & 228.9 & 313.7 & 226.7 \\
ESS Loss (MW) & 210.9 & 107 & 143.8 \\
Total Power loss (MW) & 439.8 & 420.7 & 370.5 \\
Max. VD (p.u.) & 0.0999 & 0.118 & 0.1 \\
\hline
\end{tabular}

In case 1, the simulation results were demonstrated at Figure 3(a). Some scenarios in a particular day were focused. The load demand at scenario 5 was $614.057 \mathrm{MW}$. The conventional generations and DGs were optimally generated at 478.678 MW and 139.219 MW, respectively. The total excessive powers were stored 77.780 MW as illustrated in Figure 4(a). Hence, the total power losses were 11.008 MW as illustrated in Figure 4(b). To make the point clear, scenario 25 was also focused. The load demand was $744.880 \mathrm{MW}$, whereas the conventional generations and DGs were optimally generated at 567.691MW and 182.571 MW, respectively. The total excessive powers were stored 83.4294 MW in ESS resulting the total power losses 13.116 MW as illustrated in Figure 4(a) and Figure 4(b), respectively.

In case 2 , the scenarios in a particular day were recalled from the previous case. The optimal conventional generations were totally generated at $450.806 \mathrm{MW}$, and DGs were optimally dispatched at 172.661 MW as illustrated in Figure 3(b). The total excessive powers were stored 44.338 MW in the ESS. The total power losses were 13.239 MW in scenario 5 as illustrated in Figure 4(b). In scenario 25, DGs were generated at 540.515 MW and the conventional generations were generated at $211.262 \mathrm{MW}$ to meet the optimal solutions as illustrated in Figure 3(b). The excessive powers were stored 54.738 MW leading the total power losses 11.693 MW as illustrated in Figure 4(a) and Figure 4(b). 
In proposed method, maximum renewable energy harvesting and minimum power loss were to be the objective functions. The conventional generations and DGs were optimally generated at $446.723 \mathrm{MW}$ and 172.318 MW to supply 614.057 MW of load demand in scenario 5 as illustrated in Figure 3(c). The total excessive powers were stored 44.681 MW and the total power losses were $8.8106 \mathrm{MW}$ as illustrated in Figure 4(a) and Figure 4(b). The conventional generation and DG were optimally generated at 529.986 MW and $210.093 \mathrm{MW}$ in scenario 25 to supply the load demand $744.880 \mathrm{MW}$ as illustrated in Figure 3(c). The total were stored 55.907 MW resulting the total power losses 8.703 MW as illustrated in Figure 4(a) and Figure 4(b), respectively.excessive powers.

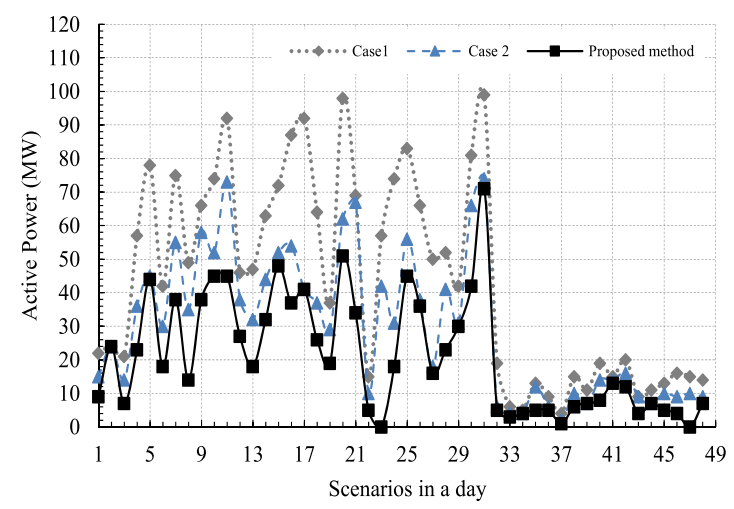

(a)

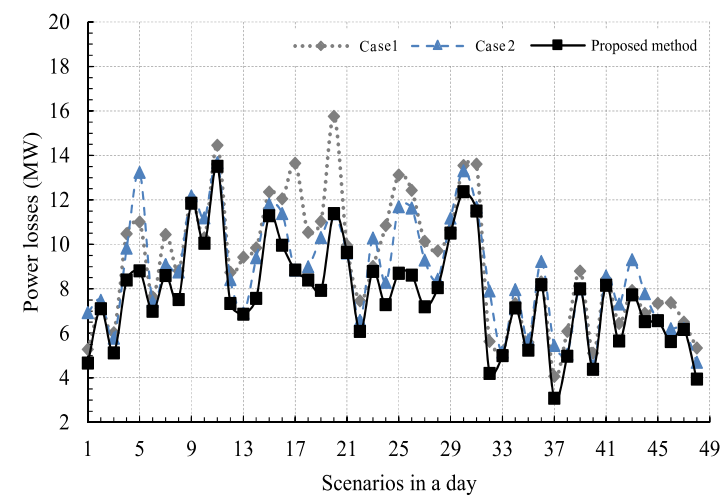

(b)

Figure 4. (a) The stored excessive power in ESS (b) Total power losses at the optimal schedule condition

The voltage profiles were extensively investigated by voltage deviation $(V D)$. Figure 5 illustrated the level of $V D$ depending on the proportion of DG in system for all cases. The worst case was obviously indicated in case 2 which the objective function of this case was maximum renewable energy harvesting. Due to the voltage constraints were addressed according to Section 2, hence the system voltages must be improved. Consequently, the proposed method was controlled the $V D$ within 0.1 p.u. during maximum renewable energy harvesting was considered. This implies that all buses in the system were still maintained within the marginal constraints where the minimum voltage is not reached below 0.95 p.u.

The rest of this simulation study aimed at the convergence rate of the proposed method. The convergence rate of proposed method is depicted in Figure 6(a). The power losses are minimized to 6 MW for scenario 46 which was approximately identified at iteration 6 . However, the proposed method was applied by changing the search space, which it did not consistent with case 1 and case 2 , for PSO algorithm to confirm its capability. At $6 \mathrm{MW}$ of total power losses, the method was approximately converged to the minimum power loss after 95 iterations as illustrated in Figure 6(b). 


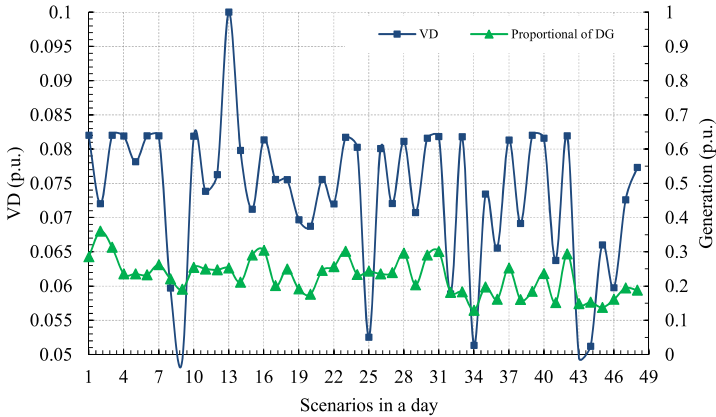

(a)

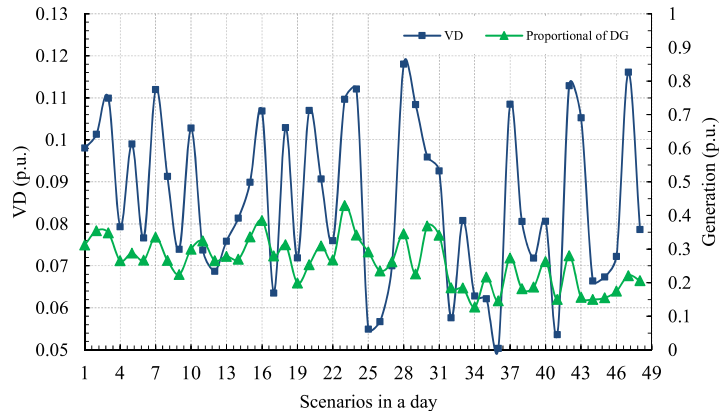

(b)

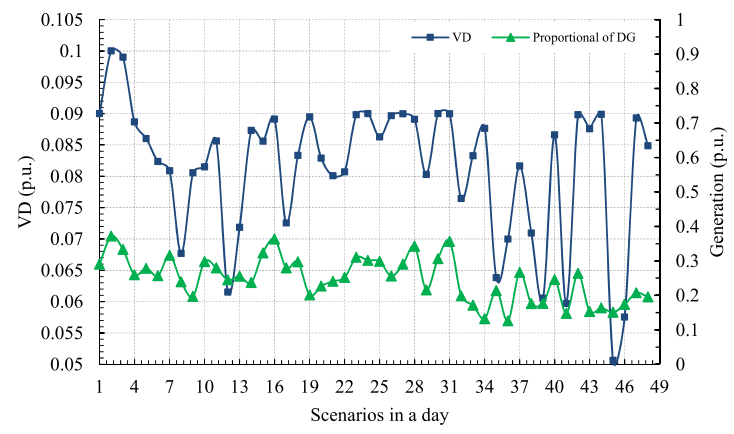

(c)

Figure 5. DG generations and voltage deviations (a) case 1 (b) case 2 (c) Proposed method

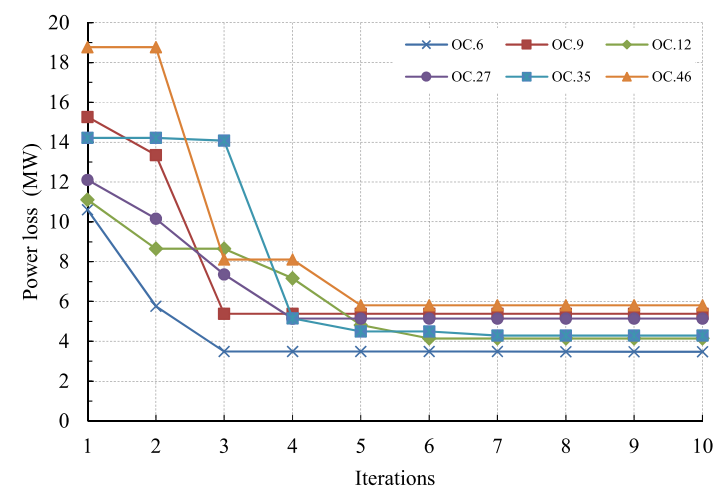

(a)

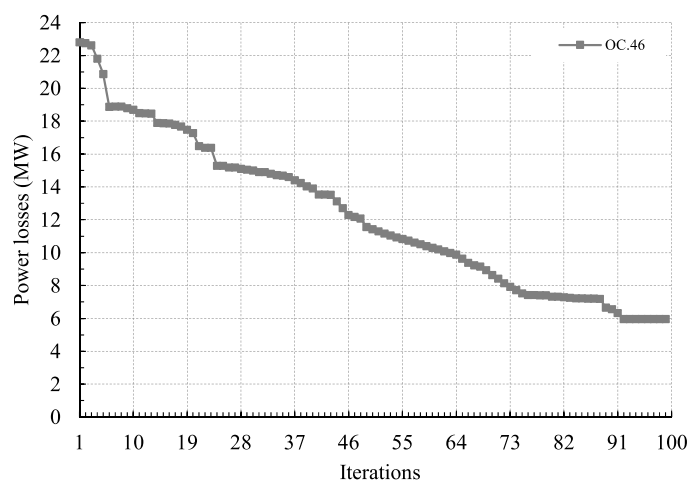

(b)

Figure 6. (a) The PSO convergence rate of case 3 for 14 bus simulation result, (b) The PSO convergence rate of scenario 46 with changing search space for 14-bus simulation result

\subsubsection{IEEE standard 30-bus system}

To confirm the capability of the proposed method, the IEEE standard 30-bus test system was employed in this section. The performed cases were still recalled for investigating the proposed method. The simulation results demonstrated the superior effectiveness and performance of the proposed method to achieve the optimal solution based on the objective functions in Section 2. The simulation results are summarized in Table 4.

The total DG dispatch over the operating scenarios in a particular day of each case studies was optimally dispatched at 436.721 MW in case 1, 588.144 MW in case 2, and 573.365 MW in proposed method case. Increasing of DG influenced the decrease in conventional generations and reducing of ESS loss. In cases 1 and 2, the generations were nevertheless dispatched with undesired solution because the total 
losses were still high as provided in Table 4, and $V D$ of the buses were reached the voltage constraints in some operating scenarios. The ESS loss was occurred as 39.509 MW in case 1, 30.260 MW in case 2, and 31.737 MW in case 3. Hence, the total power losses for optimal generation scheduling were obtained as 81.595 MW in case 1, 75.565 MW in case 2, and 65.847 MW in proposed method case as provided in Table 4.

Table 4. The IEEE 30-bus System Simulation Results

\begin{tabular}{lrrr}
\hline Parameters & Case1 & Case2 & Proposed method \\
\hline DG Harvesting (MW) & 436.721 & 588.144 & 573.365 \\
Loss in lines (MW) & 42.086 & 45.305 & 43.061 \\
ESS Loss (MW) & 39.509 & 30.260 & 31.737 \\
Total Power loss (MW) & 81.595 & 75.565 & 74.798 \\
Max. VD (p.u.) & 0.1 & 0.108 & 0.105 \\
Convergence (Iteration) & 10 & 8 & 5 \\
\hline
\end{tabular}

\subsection{Discussion}

The power delivered from the generation units to the consumer points is always accompanied with power losses. The variations of generations can directly result the power losses in the system. The trending of power losses does not only depend on the conventional generation, but available of renewable DG may also increase power losses in the system and raise the complexity of power management. Hence, power losses should be dealt with the combination of generation types in the power system.

The simulation results of comparative study demonstrate the different combination of power losses at the same operating scenarios. Although the ESS losses is obviously reduced in case 2, however, this reduction must be still included power losses in lines because the maximum dispatch of DG can result the power losses in lines especially long-distant transmission lines.

The proposed method case is to minimizing total power losses and maximizing renewable energy harvesting. Although the proposed method is allowed the higher the ESS losses than the ESS losses in case 2, the total power losses in lines are reduced by increasing some the ESS losses in a way of maximum renewable energy harvesting. In Table 3 and 4, the simulation results demonstrate that the total power losses of the proposed method is significantly lower than the cases for comparison.

The reduction of power losses are related to the optimal power for storage and minimize power loss in line. Figure 7 reveals the benefits of renewable energy harvesting and power loss reduction. This is the main advantage of the proposed method including two sections: the reduction of power losses have been improved and increased of renewable energy harvesting.

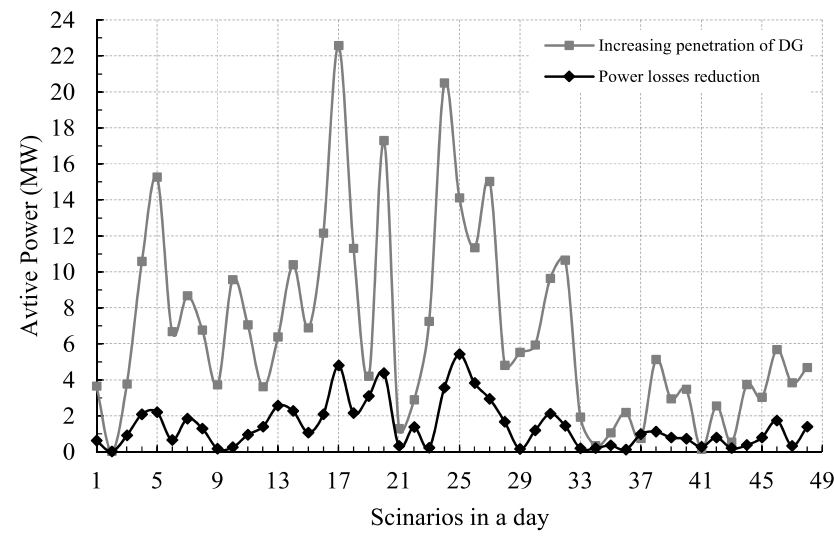

Figure 7. Relationship of power losses reduction and increasing of DG dispatch

\section{CONCLUSION}

In this paper, an optimal generation scheduling has been investigated in the power system. The proposed method was executed considering maximum renewable energy harvesting and minimizing power losses. The optimal solutions of the proposed method were identified and obtained using PSO algorithm. The two cases for comparison were performed to exploit the capability and efficiency of the 
proposed method. The simulation results demonstrated the effectiveness and performance of the proposed method to achieve the optimal solutions for generation scheduling especially with maximum renewable energy harvesting and minimizing power losses. The power losses were evidently decreased according to the optimal storing power in ESS and minimizing line losses with maximum renewable energy harvesting. Additionally, the maximum renewable energy harvesting is significantly influenced the decrease in conventional generations and reducing ESS losses.

\section{ACKNOWLEDGEMENTS}

The authors would like to thank the Princess Sirindhorn International Center for Research, Development and Technology Transfer, and the Kasetsart University scholarships for financial supports.

\section{REFERENCES}

[1] I. Serban, and C. Marinescu, "Battery energy storage system for frequency support in microgrids and with enhanced control features for uninterruptible supply of local loads," Int. J. Electr. Power Energy Syst., vol. 54, pp. 432-441, Jan. 2014.

[2] E. Vaahedi, Practical Power System Operation, New Jersey: John Wiley \& Sons Inc., 2014.

[3] S. M. Myint, and S. W. Naing, "Network reconfiguration for loss reduction and voltage profile improvement of 110-bus radial distribution system using exhaustive search techniques," International Journal of Electrical and Computer Engineering, vol. 5, no. 4, pp. 788-797, Aug. 2015

[4] H. R. Esmaeilian, and R. Fadaeinedjad, "Energy loss minimization in distribution systems utilizing an enhanced reconfiguration method integrating distributed," IEEE Syst. J., vol. 9, no. 4, pp. 1430-1439, Dec. 2015.

[5] H. Zhang, G. T. Heydt, V. Vittal, and J. Quintero, "An improved network model for transmission expansion planning considering reactive power and network losses," IEEE Trans. Power Syst., vol. 28, no. 3, pp. 3471-3479, Aug. 2013.

[6] L. Che, X. Zhang, M. Shahidehpour, A. Alabdulwahab, and A. Abusorrah, "Optimal interconnection planning of community microgrids with renewable energy sources," IEEE Trans. Smart Grid, vol. 8, no. 3, pp. 1054-1063, May 2017.

[7] A. Sadighmanesh, K. Zare, and M. Sabahi, "Distributed generation unit and capacitor placement for loss, voltage profile and ATC optimization," International Journal of Electrical and Computer Engineering, vol. 2, no. 6, pp. 774-780, Dec. 2012.

[8] J. C. Lopez, J. F. Franco, and M. J. Rider, "Optimisation-based switch allocation to improve energy losses and service restoration in radial electrical distribution systems," IET Gener. Transm. Distrib., vol. 10, no. 11, pp. 2792-2801, Aug. 2016.

[9] K. S. Ramudu, M. P. Lalitha, and P. S. Babu, "Siting and sizing of DG for loss reduction and voltage sag mitigation in RDS using ABC algorithm," International Journal of Electrical and Computer Engineering, vol. 3, no. 6, pp. 814-822, Dec. 2013.

[10] T. Gozel, and M. H. Hocaoglu, "An analytical method for the sizing and siting of distributed generators in radial systems," Elect. Power Syst. Res., vol. 79, no. 6, pp. 912-918, Jun. 2009.

[11] S. H. Win, and P. L. Swe, "Loss minimization of power distribution network using different types of distributed generation unit," International Journal of Electrical and Computer Engineering, vol. 5, no. 5, pp. 918-928, Oct. 2015.

[12] L. K. Panwar, S. R. Konda, A. Verma, B. K. Panigrahi, and R. Kumar, "Operation window constrained strategic energy management of microgrid with electric vehicle and distributed resources," IET Gener. Transm. Distrib., vol. 11 , no. 3, pp. 615-626, Feb. 2017.

[13] H. Nafisi, M. M. Agah, H. A. Abyaneh, and M. Abedi, "Two-stage optimization method for energy loss minimization in microgrid based on smart power management scheme of PHEVs," IEEE Trans. Smart Grid, vol. 7, no. 3, pp. 1268-1276, May 2016.

[14] W. Shi, N. Li, C. C. Cheng, and R. Gadh, "Real-time energy management in microgrids," IEEE Trans. Smart Grid, vol. 8, no. 1, pp. 228-238, Jan. 2017.

[15] K. G. Ing, J. J. Jamian, H. Mokhis, and H. A. Illias, "Optimum distribution network operation considering distributed generation mode of operations and safety margin,"IET Gener. Transm. Distrib., vol. 10, no. 8, pp. 1049-1058, Sep. 2016.

[16] I. K. Song, W. W. Jung, J. Y. Kim, S. Y. Yun, J. H. Choi, and S. J. Ahn, "Operation schemes of smart distribution networks with distributed energy resources for loss reduction and service restoration,"IEEE Trans. Smart Grid, vol. 4, no. 1, pp. 367-374, Mar. 2013.

[17] W. Hu, Z. Chen, B. B. Jensen, and Y. Hu, "Fuzzy adaptive particle swam optimization for power loss minimization in distribution systems using optimal load response," IET Gener. Transm. Distrib., vol. 8, no. 1, pp. 1-10, Jan. 2014.

[18] N. Nikmehr, and S. N. Ravadanegh, "Optimal operation of distributed generations in micro-grids under uncertainties in load and renerable power generation using heuristic algorithm," IET Renew. Power Gener., vol. 9, no. 8, pp. 982-990, Nov. 2015.

[19] S. Singh, M. Singh, and S. C. Kaushik, "Optimal power scheduling of renewable energy systems in microgrids using distributed energy storage system,” IET Renew. Power Gener., vol. 10, no. 9, pp. 1328-1339, Oct. 2016. 
[20] K. Panagiotou, C. Klumpner, M. Sumner, "The effect of including power converter losses when modelling energy storage systems: A UK domestic study," EPE'16 ECCE Europe, 2016.

[21] J. Grainger, and W. Stevenson, Power System Analysis, McGraw-Hill, Jan. 1994.

[22] A. P. Engelbrecht, Fundamentals of Computational Swarm Intelligence, Hoboken, NJ, USA:Wiley, 2006.

[23] D. Bratton, J. Kennedy, "Defining a standard for particle swarm optimization," Proc. Swarm Intell. Symp., pp. 120-127, 2007.

\section{BIOGRAPHIES OF AUTHORS}

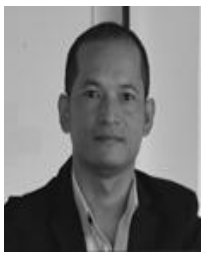

Bonthanh Banhthasit received the B.Eng. degree in electrical engineering from National Univesity Of Laos (NUOL) in 2001. He received M.Eng in electrical engineering from Khon Kean University, Thailand in 2011. Currently, he is a Ph.D. student at the Kasetsart University, Thailand. His research interests are power system operation and optimization, and breakdown of high voltage insulation.

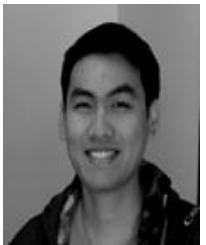

Chaowanan Jamroen received the B.Eng. and M.Eng. degrees from Kasetsart Unversity, Bangkok, Thailand, in 2013, and 2016, respectively, all in electrical engineering. He is currently a lecturer at Division of Instrumentation and Automation Engineering Technology, King Mongkut's University of Technology North Bangkok, Rayong Campus, Thailand. His research interests include power system analysis, power system stability and control, smart grid technology, synchrophasor measurement technique, power system optimization, and distributed generation.

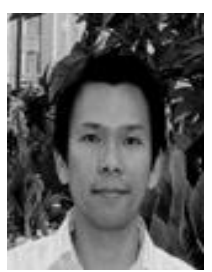

Sanchai Dechanupaprittha received his B.Eng. and M.Sc. (Electrical Engineering) from Sirindhorn International Institute of Technology (SIIT), Thammasat University, Thailand in 2000 and 2003, respectively. And he received his D.Eng. (Electrical Engineering) from Kyushu Institute of Technology (Kyutech), Japan in 2008. He is currently an assistant professor at Kasetsart University. His research interests are power system stability analysis, dynamics and robust control, power system optimization and computational intelligence, wide-area monitoring, protection and control, and smart grids and energy efficiency. 